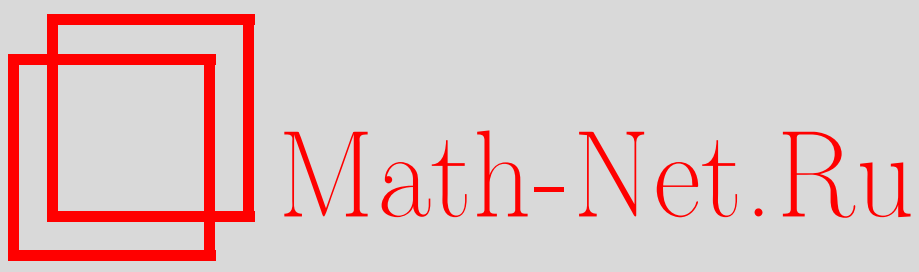

V. V. Volchkov, Vit. V. Volchkov, Analogs of the liouville property for harmonic functions on unbounded domains, Sibirsk. Mat. Zh., 2020, Volume 61, Number 4, 752 764

DOI: https://doi.org/10.33048/smzh.2020.61.403

Use of the all-Russian mathematical portal Math-Net.Ru implies that you have read and agreed to these terms of use http://www . mathnet.ru/eng/agreement

Download details:

IP: 3.85 .73 .92

April 26, 2023, 17:45:20

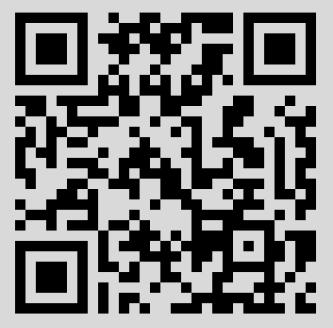


Сибирский математический журнал Июль-август, 2020. Том 61, № 4

УДК 517.5

\title{
АНАЛОГИ СВОЙСТВА ЛИУВИЛЛЯ ДЛЯ ГАРМОНИЧЕСКИХ ФУНКЦИЙ НА НЕОГРАНИЧЕННЫХ ОБЛАСТЯХ
}

\section{В. В. Волчков, Вит. В. Волчков}

\begin{abstract}
Аннотация. Получены аналоги свойства Лиувилля для функций, гармонических на внешности жордановой области $G \subset \mathbb{C}$ и имеющих постоянные граничные значения для самой функции и ее нормальной производной. Показано, что в общем случае указанные условия ослабить нельзя.
\end{abstract}

DOI 10.33048/smzh.2020.61.403

Ключевые слова: свойство Лиувилля, гармонические функции, граничное поведение.

\section{Введение}

Известно, что ограниченная аналитическая функция на комплексной плоскости $\mathbb{C}$ постоянна. Этот факт называется свойством Лиувилля для аналитических функций. В указанном утверждении аналитические функции можно заменить гармоническими, а плоскость $\mathbb{C}$ - произвольным евклидовым пространством $\mathbb{R}^{n}$. Одна из первых теорем такого вида утверждает (см. $[1, \S 24$, п. 11]), что если функция $f$ является гармонической в $\mathbb{R}^{n}$ и ограничена сверху (или снизу), то она постоянна. Еще одна теорема является следствием более общего результата из $[2$, ч. 3, гл. $3, \S 3.2]$ и касается функций, гармонических на внешности компакта в $\mathbb{R}^{n}$ со связным дополнением. Эта теорема утверждает, что если такая функция убывает при $|x| \rightarrow \infty$ быстрее любой отрицательной степени $|x|$ (здесь $|x|$ - евклидова норма), то она нулевая, при этом указанную скорость убывания ослабить нельзя.

В некоторых утверждениях такого типа можно ослабить условие гармоничности. В частности, изучалось поведение на бесконечности для функций, удовлетворяющих уравнениям средних значений по сферам (или шарам) с ограничениями на рассматриваемые множества центров и радиусов [2-5]. Например, в $[2$, ч. 5, гл. $5 ; 4]$ получены точные теоремы типа Лиувилля для функций в $\mathbb{R}^{n}$, удовлетворяющих теореме о среднем для всех центров и одного фиксированного радиуса. Можно показать (см. [2, ч. 5, гл. 5]), что данный класс функций значительно шире класса гармонических в $\mathbb{R}^{n}$ функций. Отметим также интересный результат Хансена $[5,6]:$ если $f$ - непрерывная ограниченная функция на $\mathbb{R}^{2}$ такая, что для любого $x \in \mathbb{R}^{2}$

$$
f(x)=\frac{1}{2 \pi} \int_{0}^{2 \pi} f\left(x+r(x) e^{i t}\right) d t,
$$

(с) 2020 Волчков В. В., Волчков Вит. В. 
где $r(x)$ - положительная функция на $\mathbb{R}^{2}$, удовлетворяющая условию

$$
\varlimsup_{x \rightarrow \infty}(r(x)-|x|)<+\infty,
$$

то $f$ - константа.

Многие авторы исследовали свойство Лиувилля для решений некоторых дифференциальных уравнений и неравенств на различных пространствах (см. [7-11] и библиографию в них).

Еще одним важным направлением в рассматриваемой тематике является изучение возможного асимптотического поведения на бесконечности периодических в среднем функций. Очевидным свойством ненулевых периодических функций на вещественной оси является невозможность их стремления к нулю на бесконечности. В многомерном случае, где возможны различные обобщения понятия периодичности, ситуация становится значительно сложнее. Развивая теорию периодических в среднем функций, Йон [12, гл. 6] получил следующий результат: если непрерывная функция $f$ на $\mathbb{R}^{3}$ имеет нулевые интегралы по всем сферам единичного радиуса и

$$
f(x)=o\left(\frac{1}{|x|}\right) \quad \text { при } x \rightarrow \infty,
$$

то $f \equiv 0$ (см. также [13], где рассмотрен $n$-мерный случай). Примеры показывают (см. $[12,13])$, что указанное условие убывания функции $f$ точное. Теорема Йона получила дальнейшее развитие и уточнение в разных направлениях [14$23]$.

Во-первых, изучались ее обобщения для функций $f$, удовлетворяющих уравнению свертки $f * T=0$, где $T$ - заданное ненулевое распределение в $\mathbb{R}^{n}$ с компактным носителем. Выяснилось, в частности, что не существует ненулевых решений $f$, принадлежащих $L^{p}\left(\mathbb{R}^{n}\right)$ при некотором $p \in[1,2 n /(n-1)], n \geq 2$. При этом показатель $p$ не может быть увеличен [14-17].

Во-вторых, доказаны так называемые «спектральные» аналоги теоремы Йона. Как и в классической теореме Лиувилля для целых функций, в них установлена связь между поведением на бесконечности решений уравнений свертки и множеством нулевых коэффициентов в ее разложении Фурье по сферическим гармоникам [2, ч. 3 , гл. $3 ; 18$, ч. 3 , гл. 14].

В-третьих, исследовалась задача о допустимом росте (убывании) функций с нулевыми сферическими средними на неограниченных областях. Здесь возникли интересные эффекты, связанные с зависимостью от вида области [2, ч 3 , гл. $3, \S 3.3 ; 18$, ч. 3 , гл. $14, \S 14.6 ; 19$, ч. 2 , гл. $1, \S 1.6]$. К рассматриваемому кругу идей относятся также различные теоремы типа Фрагмена - Линделёфа и теоремы о граничном поведении аналитических функций [20,21].

В-четвертых, получены аналоги теоремы Йона на симметрических пространствах [18, ч. 3, гл. 15; 22]. Разработанные для этого методы оказались весьма полезными при изучении близких вопросов для искаженного уравнения свертки на комплексном евклидовом пространстве и группе Гейзенберга [23].

В данной работе получены аналоги свойства Лиувилля для функций, гармонических на внешности жордановой области $G \subset \mathbb{C}$ и имеющих постоянные граничные значения для самой функции и ее нормальной производной (см. теоремы 1 и 2 ниже). Кроме того, показано, что в общем случае условия теоремы 1 нельзя ослабить (см. теорему 3 ). 


\section{$\S 1$. Формулировки основных результатов}

Пусть $\Gamma$ - замкнутая гладкая жорданова кривая в комплексной плоскости $\mathbb{C}, G$ - ограниченная область в $\mathbb{C}$ с границей $\Gamma, \bar{G}=G \cup \Gamma$. На кривой $\Gamma$ стандартным образом вводится мера $\mu$, которая на каждом открытом подмножестве Г равна сумме длин составляющих его дуг (см. [24, гл. 10, §1]).

Как обычно, символом $\frac{\partial}{\partial n}$ будем обозначать оператор дифференцирования в направлении внешней (по отношению к $G$ ) нормали к $\Gamma$.

Для функции $f$, непрерывной на окружности $\gamma_{r}=\{z \in \mathbb{C}:|z|=r\}$, положим

$$
M_{r}(f)=\int_{\gamma_{r}}|f(z)||d z| .
$$

Сформулируем основные результаты данной работы.

Теорема 1. Пусть функция $f \in C(\mathbb{C} \backslash G)$ гармонична в $\mathbb{C} \backslash \bar{G}$ и для некоторых $c_{1}, c_{2} \in \mathbb{C}$ выполнены следующие условия:

(1) $f=c_{1}$ на $\Gamma$;

(2) $\frac{\partial f}{\partial n}=c_{2} \mu$-почти всюду на Г;

(3) $\varlimsup_{r \rightarrow \infty} \frac{1}{r^{3}} M_{r}(f)=0$.

Тогда если $G$ не является кругом, то $f=c_{1}$ в $\mathbb{C} \backslash G$. Если $G-$ круг, то

$$
f(z)=c_{1}+c_{2} R \ln \frac{\left|z-z_{0}\right|}{R}, \quad z \in \mathbb{C} \backslash G,
$$

где $z_{0}, R$ - центр и радиус круга $G$.

Доказательство теоремы 1 основано на применении конформного отображения внешности единичного круга на область $\mathbb{C} \backslash \bar{G}$. Это отображение позволяет свести исходную задачу к переопределенной краевой задаче для уравнения Лапласа на внешности круга, в которой основной трудностью является неоднородность граничного условия для нормальной производной. Для изучения этого условия потребовались некоторые тонкие результаты о граничных свойствах функции, осуществляющей указанное выше конформное отображение, а также некоторые свойства классов Харди $H_{p}$ в единичном круге (см. [24, гл. 9, 10]). Соответствующие вспомогательные конструкции и утверждения содержатся в $\S 2$. Отметим, что отсутствие соответствующего аппарата в многомерном случае оставляет открытым вопрос о существовании аналога теоремы 1 в пространстве $\mathbb{R}^{n}$ при $n>2$.

Из теоремы 1 сразу вытекает

Следствие 1. Пусть функция $g \in C(\mathbb{C} \backslash G)$ голоморфна в $\mathbb{C} \backslash \bar{G}$ и для функции $f=\operatorname{Re} g$ выполнены условия 1-3 теоремы 1 . Тогда $g-$ константа.

Далее, если в теореме 1 положить $c_{2}=0$, то получим, что функция $f$ обязана быть тождественно постоянной независимо от вида $G$. Как показывает следующая теорема, данное утверждение сохраняет силу и при более слабых предположениях.

Теорема 2. Пусть функция $f \in C(\mathbb{C} \backslash G)$ гармонична в $\mathbb{C} \backslash \bar{G}$ и для некоторого $c \in \mathbb{C}$ выполнены следующие условия:

(1) $f=c$ на $\Gamma$;

(2) $\frac{\partial f}{\partial n}=0$ на некотором множестве $E \subset$ Г положительной меры $\mu$;

(3) $\varlimsup_{r \rightarrow \infty} \frac{1}{r^{\alpha}} M_{r}(f)=0$ для некоторого $\alpha>0$. 
Тогда $f=c$ в $\mathbb{C} \backslash G$.

Следующий результат показывает необходимость условий 1,2 и точность условия 3 в теореме 1.

Теорема 3. Существуют отличная от круга ограниченная центральносимметричная область $G \subset \mathbb{C}$ с гладкой жордановой границей $\Gamma$ и функции $f_{1}, f_{2}, f_{3}$, непрерывные в $\mathbb{C} \backslash G$ и гармонические в $\mathbb{C} \backslash \bar{G}$, такие, что

(1) $f_{1}$ удовлетворяет условиям (1) и (3) теоремы 1 и не является тождественной константой;

(2) $f_{2}$ удовлетворяет условию (3) теоремы 1 и

$$
\frac{\partial f_{2}}{\partial n}=1 \quad \text { всюду на } Г
$$

(3) $f_{3}=0$ и $\frac{\partial f_{3}}{\partial n}=1$ всюду на Г, при этом

$$
f_{3}(z)=O\left(|z|^{2}\right) \quad \text { при } z \rightarrow \infty \text {. }
$$

$B$ частности, $M_{r}\left(f_{3}\right)=O\left(r^{3}\right)$ при $r \rightarrow \infty$.

Доказательства теорем 1-3 содержатся в $\S 3$.

\section{§ 2. Вспомогательные конструкции и утверждения}

Пусть $A=\{z \in \mathbb{C}:|z|>1\}$ и функция $u$ непрерывна в $\bar{A}=\{z \in \mathbb{C}:|z| \geq 1\}$. При любом фиксированном $\rho \geq 1$ функции $u\left(\rho e^{i \varphi}\right)$ соответствует ряд Фурье

$$
u\left(\rho e^{i \varphi}\right)=\sum_{n=-\infty}^{\infty} u_{n}(\rho) e^{i n \varphi}, \quad \varphi \in[0,2 \pi]
$$

где

$$
u_{n}(\rho)=\frac{1}{2 \pi} \int_{0}^{2 \pi} u\left(\rho e^{i \varphi}\right) e^{-i n \varphi} d \varphi .
$$

Пусть $\Delta=\frac{\partial^{2}}{\partial x^{2}}+\frac{\partial^{2}}{\partial y^{2}}-$ оператор Лапласа. Если $u \in C^{2}(A)$, то простые вычисления с использованием (4) показывают, что при любых $\rho>1, \varphi \in[0,2 \pi]$, $n \in \mathbb{Z}$ выполнено равенство

$$
\Delta\left(u_{n}(\rho) e^{i n \varphi}\right)=\frac{1}{2 \pi} \int_{0}^{2 \pi}(\Delta u)\left(\rho e^{i t}\right) e^{i n(\varphi-t)} d t .
$$

Кроме того,

$$
\Delta\left(u_{n}(\rho) e^{i n \varphi}\right)=\left(u_{n}^{\prime \prime}(\rho)+\frac{u_{n}^{\prime}(\rho)}{\rho}-\frac{n^{2}}{\rho^{2}} u_{n}(\rho)\right) e^{i n \varphi} .
$$

По теореме Римана о конформном отображении существует единственная голоморфная в области $A$ функция $w=\psi(z)$, которая отображает $A$ конформно на область $\mathbb{C} \backslash G$ при условиях

$$
\psi(\infty)=\infty, \quad \psi^{\prime}(\infty)=a>0 .
$$

Первое условие в (7) показывает, что функция $w=\psi(z)$ точку $z=\infty$ переводит в точку $w=\infty$, а второе условие означает, что

$$
\lim _{z \rightarrow \infty} \frac{\psi(z)}{z}=a>0 .
$$


Из условий (7) и (8) следует, что функция $\psi$, являясь голоморфной в области $A$, имеет в точке $z=\infty$ простой полюс, поэтому ее лорановское разложение в $A$ имеет вид

$$
\psi(z)=a z+\sum_{0}^{\infty} \psi_{n} z^{-n}, \quad a>0 .
$$

Согласно принципу соответствия границ при конформных отображениях функция $\psi$ может быть продолжена по непрерывности на множество $\bar{A}$. Для этого продолжения сохраним обозначение $w=\psi(z)$.

Пусть $\mathbb{D}=\{z \in \mathbb{C}:|z|<1\}, \mathbb{T}=\{z \in \mathbb{C}:|z|=1\}$. Можно показать (см. [24, гл. $10, \S 1$, теорема 2$]$ ), что любому множеству положительной лебеговой меры на $\mathbb{T}$ при отображении $\psi$ соответствует $\mu$-измеримое множество положительной меры на $\Gamma$, и обратно. Аналогично всякому множеству меры нуль на $\mathbb{T}$ соответствует множество точек нулевой меры $\mu$ на $Г$, и обратно.

Как обычно, обозначим через $H_{p}(\mathbb{D}), p>0$, класс функций $f$, голоморфных в $\mathbb{D}$ и таких, что для каждой из них интеграл

$$
\int_{0}^{2 \pi}\left|f\left(r e^{i \varphi}\right)\right|^{p} d \varphi
$$

ограничен при $0 \leq r<1$. При $z \in \mathbb{D}$ положим

$$
h(z)=a-\sum_{0}^{\infty} n \psi_{n} z^{-n-1}=\psi^{\prime}\left(\frac{1}{z}\right) .
$$

Отметим, что в силу однолистности $\psi$ функция $h$ не имеет нулей в $\mathbb{D}$. Далее потребуются некоторые вспомогательные утверждения о свойствах функций $h$ и $\psi$.

Лемма 1. Функции $h$ и $1 / h$ принадлежат классу $H_{p}(\mathbb{D})$ при всех $p>0$.

ДоказАтельство. Пусть $b \in G$. Обозначим через $d(b)$ расстояние от точки $b$ до Г. Тогда при любом $z \in \mathbb{D}$ имеем

$$
\left|\psi\left(\frac{1}{z}\right)-b\right| \geq d(b)>0 .
$$

Из этого неравенства и (9) следует, что существует постоянная $c_{1}>0$ такая, что

$$
\left|z\left(\psi\left(\frac{1}{z}\right)-b\right)\right|>c_{1}
$$

при всех $z \in \mathbb{D}$. Кроме того, из (9) и определения $\psi$ получаем, что

$$
\left|z\left(\psi\left(\frac{1}{z}\right)-b\right)\right|<c_{2}, \quad z \in \mathbb{D},
$$

где $c_{2}>0$ не зависит от $z$. Далее, при $z \in \mathbb{D}$ из (11) находим

$$
h(z)=-z^{2}\left(\psi\left(\frac{1}{z}\right)-b\right)^{2} \lambda^{\prime}(z),
$$

где

$$
\lambda(z)=\left(\psi\left(\frac{1}{z}\right)-b\right)^{-1} .
$$


Учитывая оценки (12) и (13), при любых $p>0, r \in[0,1)$ из (14) получаем

$$
\begin{aligned}
\int_{0}^{2 \pi}\left(\left|h\left(r e^{i \varphi}\right)\right|^{p}+\left|h\left(r e^{i \varphi}\right)\right|^{-p}\right) & d \varphi \\
& <c_{2}^{2 p} \int_{0}^{2 \pi}\left|\lambda^{\prime}\left(r e^{i \varphi}\right)\right|^{p} d \varphi+c_{1}^{-2 p} \int_{0}^{2 \pi}\left|\lambda^{\prime}\left(r e^{i \varphi}\right)\right|^{-p} d \varphi .
\end{aligned}
$$

Функция $\lambda$ отображает круг $\mathbb{D}$ конформно и однолистно на некоторую ограниченную область с гладкой жордановой границей. По теореме Линделёфа (см. [24, гл. 10, §1, теорема 4]) при надлежащем выборе ветви аргумента функции $\arg \lambda^{\prime}(z)$ и $\arg 1 / \lambda^{\prime}(z)$ могут быть продолжены до непрерывных функций на $\overline{\mathbb{D}}$. Отсюда следует (см. $[24$, гл. $10, \S 1$, теорема 5]), что функции $\lambda$ и $1 / \lambda$ принадлежит классу $H_{p}(\mathbb{D})$ при любом $p>0$. Таким образом, интегралы в правой части неравенства (15) ограничены по $r$. Отсюда и из (15) получаем утверждение леммы 1.

Следствие 2. Функция $h$ имеет почти всюду на $\mathbb{T}$ конечные предельные значения по некасательным путям, образующие граничную функцию $h\left(e^{i \varphi}\right)$, $\varphi \in(0,2 \pi)$. При этом $h\left(e^{i \varphi}\right) \in L^{p}(0,2 \pi)$ для любого $p>0$ и

$$
h\left(e^{i \varphi}\right) \neq 0 \quad \text { для почти всех } \varphi \in(0,2 \pi) .
$$

ДоКАЗАТЕЛЬСТво. Утверждение о существовании указанной предельной функции $h\left(e^{i \varphi}\right)$ и ее принадлежности классу $L^{p}(0,2 \pi)$ следует из леммы 1 и известного аналогичного свойства для любой функции класса $H_{p}(\mathbb{D})($ см., например, $[24$, гл. $9, \S 4])$. Применяя данное свойство к функции $1 / h$ и используя лемму 1 , получим (16).

Следствие 3. Пусть $\alpha, \beta \in \mathbb{C}$ и $|\alpha| \geq|\beta|>0$. Тогда функция

$$
h_{1}(z)=\frac{h(z)}{(\alpha+\beta z)^{2}}
$$

принадлежит классу $H_{p}(\mathbb{D})$ при всех $0<p<\frac{1}{4}$.

ДокАзАтЕльство. Сначала предположим, что $|\beta|=|\alpha|$. В этом случае $\alpha+\beta z=\beta\left(z-e^{i \gamma}\right)$ при некотором $\gamma \in[0,2 \pi]$. Пусть $0<p<\frac{1}{4}$. Выберем $q>1$ так, что $q p<\frac{1}{4}$. Используя неравенство Гёльдера и лемму 1 , для любого $r \in[0,1)$ имеем

$$
\begin{aligned}
\int_{0}^{2 \pi}\left|h_{1}\left(r e^{i \varphi}\right)\right|^{p} d \varphi=\int_{0}^{2 \pi}\left|h\left(r e^{i \varphi}\right)\right|^{p}\left|\beta\left(r e^{i \varphi}-e^{i \gamma}\right)\right|^{-2 p} d \varphi & \\
\leq\left(\int_{0}^{2 \pi}\left|h\left(r e^{i \varphi}\right)\right|^{\frac{p q}{q-1}} d \varphi\right)^{\frac{q-1}{q}} & \left(\int_{0}^{2 \pi}\left|\beta\left(r e^{i \varphi}-e^{i \gamma}\right)\right|^{-2 p q} d \varphi\right)^{\frac{1}{q}} \\
& \leq c\left(\int_{0}^{2 \pi}\left|1-r e^{i(\varphi-\gamma)}\right|^{-2 p q} d \varphi\right)^{\frac{1}{q}},
\end{aligned}
$$


где постоянная $c>0$ не зависит от $r$. Поскольку

$$
\left|1-r e^{i(\varphi-\gamma)}\right| \geq 1-|\cos (\varphi-\gamma)|
$$

и $p q<\frac{1}{4}$, из (17) получаем требуемое утверждение. В случае $|\alpha|>|\beta|>0$ утверждение очевидно в силу леммы 1 и неравенства $|\alpha+\beta z| \geq|\alpha|-|\beta|, z \in$ $\mathbb{D}$.

Лемма 2. Для почти всех (по мере Лебега) $\varphi \in[0,2 \pi]$ имеет место равенство

$$
\lim _{\varepsilon \rightarrow+0} \psi^{\prime}\left((1+\varepsilon) e^{i \varphi}\right)=\lim _{\varepsilon \rightarrow+0} \frac{\psi\left((1+\varepsilon) e^{i \varphi}\right)-\psi\left(e^{i \varphi}\right)}{\varepsilon e^{i \varphi}},
$$

где оба предела существуют и конечны.

ДокаЗАТЕЛЬСтво. Из (11) имеем $\psi^{\prime}\left((1+\varepsilon) e^{i \varphi}\right)=h\left((1+\varepsilon)^{-1} e^{-i \varphi}\right)$ для любых $\varepsilon>0, \varphi \in[0,2 \pi]$. Отсюда и из следствия 2 вытекает существование конечного предела в левой части равенства (18) для почти всех $\varphi \in[0,2 \pi]$. По теореме о среднем для таких $\varphi$ выполнено равенство

$$
\int_{1}^{1+\varepsilon} \psi^{\prime}\left(\rho e^{i \varphi}\right) d \rho=\varepsilon \psi^{\prime}\left(\xi e^{i \varphi}\right)
$$

для любого $\varepsilon>0$ и некоторого $\xi \in(1,1+\varepsilon)$, зависящего от $\varepsilon$. Следовательно, существует конечный предел $\lim _{\varepsilon \rightarrow+0} \frac{1}{\varepsilon} \int_{1}^{1+\varepsilon} \psi^{\prime}\left(\rho e^{i \varphi}\right) d \rho$, который равен пределу в левой части равенства (18). Поскольку

$$
\int_{1}^{1+\varepsilon} \psi^{\prime}\left(\rho e^{i \varphi}\right) d \rho=\frac{\psi\left((1+\varepsilon) e^{i \varphi}\right)-\psi\left(e^{i \varphi}\right)}{e^{i \varphi}},
$$

отсюда получаем утверждение леммы 2.

Лемма 3. Для почти всех $\varphi \in(0,2 \pi)$ существует $\delta=\delta(\varphi)>0$ такое, что при любом $\varepsilon \in(0, \delta(\varphi))$ круг

$$
K_{\varepsilon, \varphi}=\left\{z \in \mathbb{C}:\left|z-\psi\left(e^{i \varphi}\right)-\varepsilon e^{i \varphi} \psi^{\prime}\left(e^{i \varphi}\right)\right| \leq \frac{\varepsilon}{2}\left|\psi^{\prime}\left(e^{i \varphi}\right)\right|\right\}
$$

не пересекается с $Г$.

ДокАЗАтЕЛЬСтво. Предположим, что для некоторого $\varphi \in(0,2 \pi)$ существует последовательность $\left\{\varepsilon_{j}\right\}_{j=1}^{\infty}$ положительных чисел такая, что $\varlimsup_{j \rightarrow \infty} \varepsilon_{j}=0$ и при любом $j$ круг $K_{\varepsilon_{j}, \varphi}$ пересекается с $Г$. Обозначим через $\varphi_{j}$ одну из точек полуинтервала $[0,2 \pi)$, для которой $\psi\left(e^{i \varphi_{j}}\right) \in K_{\varepsilon_{j}, \varphi} \cap \Gamma$. Тогда имеем

$$
\left|\psi\left(e^{i \varphi_{j}}\right)-\psi\left(e^{i \varphi}\right)-\varepsilon_{j} e^{i \varphi} \psi^{\prime}\left(e^{i \varphi}\right)\right| \leq \frac{\varepsilon_{j}}{2}\left|\psi^{\prime}\left(e^{i \varphi}\right)\right| .
$$

Из этого неравенства и однолистности $\psi$ заключаем, что $\varphi_{j} \rightarrow \varphi$ при $j \rightarrow \infty$.

Далее, функция $\psi\left(e^{i t}\right)$ абсолютно непрерывна на $[0,2 \pi]$ и для почти всех $t \in[0,2 \pi]$ выполнено равенство

$$
\frac{d}{d t} \psi\left(e^{i t}\right)=i e^{i t} \psi^{\prime}\left(e^{i t}\right)
$$

(см. $[24$, гл. $10, \S 1$, теорема 1]). 
Предположим теперь, что $\psi^{\prime}\left(e^{i \varphi}\right) \neq 0$ и

$$
\psi\left(e^{i \varphi}\right)-\psi\left(e^{i \varphi_{j}}\right)=i \psi^{\prime}\left(e^{i \varphi}\right) e^{i \varphi}\left(\varphi-\varphi_{j}\right)+o\left(\varphi-\varphi_{j}\right) \quad \text { при } j \rightarrow \infty .
$$

Из следствия 2 и равенства (21) следует, что эти требования выполняются для почти всех $\varphi \in(0,2 \pi)$. Сопоставляя $(22)$ и $(20)$ и учитывая, что $\psi^{\prime}\left(e^{i \varphi}\right) \neq 0$, приходим к неравенству

$$
\left|\varepsilon_{j}+i\left(\varphi-\varphi_{j}\right)+o\left(\varphi-\varphi_{j}\right)\right| \leq \frac{\varepsilon_{j}}{2} \quad \text { при } j \rightarrow \infty .
$$

При достаточно больших $j$ последнее неравенство противоречиво, откуда следует утверждение леммы 3.

Лемма 4. Пусть $f$ гармонична в круге $K=\left\{\zeta \in \mathbb{C}:\left|\zeta-\zeta_{0}\right|<r\right\}$ и

$$
M=\sup _{\zeta \in K}|f(\zeta)|<+\infty .
$$

Тогда при любом $\zeta \in K$ имеет место оценка

$$
\left|f(\zeta)-f\left(\zeta_{0}\right)\right| \leq \frac{2 M\left|\zeta-\zeta_{0}\right|}{r-\left|\zeta-\zeta_{0}\right|}
$$

ДокАЗАТЕЛЬСТво. Из условия леммы получаем, что $w(z)=f\left(r z+\zeta_{0}\right)$ гармонична в $\mathbb{D}$ и $|w(z)| \leq M$ для любого $z \in \mathbb{D}$. Отсюда следует (см., например, [24, гл. $9, \S 2$, следствие 2$]$ ), что почти всюду на $\mathbb{T}$ функция $w$ имеет некасательные конечные предельные значения. Как обычно, сохраним обозначение $w\left(e^{i t}\right)$ для соответствующей предельной функции, определенной почти всюду на $\mathbb{T}$. Тогда из (23) имеем $\left|w\left(e^{i t}\right)\right| \leq M$. Кроме того, для любого $z=\rho e^{i \varphi}, 0 \leq \rho<1$, выполнена формула Пуассона

$$
w(z)-w(0)=\frac{1}{\pi} \int_{0}^{2 \pi} w\left(e^{i t}\right) \sum_{n=1}^{\infty} \rho^{n} \cos (n(\varphi-t)) d t
$$

(см. [24, гл. 9, §2, теорема 3]). Интеграл справа не превосходит выражения

$$
\frac{1}{\pi} \int_{0}^{2 \pi}\left|w\left(e^{i t}\right)\right| \sum_{n=1}^{\infty} \rho^{n} d t \leq \frac{2 M \rho}{1-\rho},
$$

поэтому оценка (24) следует из (25).

Лемма 5. Для почти всех $\varphi \in(0,2 \pi)$ выполнено следующее утверждение: если $f$ гармонична в $\mathbb{C} \backslash \bar{G}$, непрерывна в $\mathbb{C} \backslash G$ и существует производная $\frac{\partial f}{\partial n}\left(\psi\left(e^{i \varphi}\right)\right)$, то

$$
\lim _{\varepsilon \rightarrow+0} \frac{f\left(\psi\left((1+\varepsilon) e^{i \varphi}\right)\right)-f\left(\psi\left(e^{i \varphi}\right)\right)}{\varepsilon}=\frac{\partial f}{\partial n}\left(\psi\left(e^{i \varphi}\right)\right)\left|\psi^{\prime}\left(e^{i \varphi}\right)\right| .
$$

ДокАЗАТЕЛЬСтво. Из следствия 2 и леммы 2 вытекает, что для почти всех $\varphi \in(0,2 \pi)$ существует отличный от нуля конечный предел в левой части равенства (18) и

$$
\psi\left((1+\varepsilon) e^{i \varphi}\right)=\psi\left(e^{i \varphi}\right)+\varepsilon e^{i \varphi} \psi^{\prime}\left(e^{i \varphi}\right)+o(\varepsilon) \quad \text { при } \varepsilon \rightarrow+0 .
$$


Следовательно, для таких $\varphi$ точка $\psi\left((1+\varepsilon) e^{i \varphi}\right)$ содержится в круге $K_{\varepsilon, \varphi}$ (см. (19)) при всех достаточно малых $\varepsilon>0$. Применим лемму 4 , полагая

$$
\zeta_{0}=\psi\left(e^{i \varphi}\right)+\varepsilon e^{i \varphi} \psi^{\prime}\left(e^{i \varphi}\right), \quad r=\frac{\varepsilon}{2}\left|\psi^{\prime}\left(e^{i \varphi}\right)\right|, \quad \zeta=\psi\left((1+\varepsilon) e^{i \varphi}\right),
$$

где $\varepsilon \in(0, \delta(\varphi))$ достаточно мало. Поскольку круг $K_{\varepsilon, \varphi}$ содержится в круге $\left\{z \in \mathbb{C}:|z| \leq\left|\psi\left(e^{i \varphi}\right)\right|+\frac{3}{2} \delta(\varphi)\left|\psi^{\prime}\left(e^{i \varphi}\right)\right|\right\}$, существует константа $M_{\varphi}$, не зависящая от $\varepsilon$, такая, что

$$
\sup _{K_{\varepsilon, \varphi}}|f| \leq M_{\varphi} \quad \text { для всех } \varepsilon \in(0, \delta(\varphi)) .
$$

Тогда из (26) и леммы 4 получаем

$$
f\left(\psi\left((1+\varepsilon) e^{i \varphi}\right)\right)-f\left(\psi\left(e^{i \varphi}\right)+\varepsilon e^{i \varphi} \psi^{\prime}\left(e^{i \varphi}\right)\right)=o(\varepsilon) \quad \text { при } \varepsilon \rightarrow+0 .
$$

Из определения нормальной производной имеем

$$
\frac{\partial f}{\partial n}\left(\psi\left(e^{i \varphi}\right)\right)=\lim _{\varepsilon \rightarrow+0} \frac{f\left(\psi\left(e^{i \varphi}\right)+\varepsilon e^{i \varphi} \psi^{\prime}\left(e^{i \varphi}\right)\right)-f\left(\psi\left(e^{i \varphi}\right)\right)}{\varepsilon\left|\psi^{\prime}\left(e^{i \varphi}\right)\right|} .
$$

Из этого равенства и соотношения (27) следует утверждение леммы 5.

Лемма 6. Пусть $f$ гармонична в $\mathbb{C} \backslash G$ и

$$
\varlimsup_{r \rightarrow \infty} \frac{M_{r}(f)}{r^{\alpha}}=0
$$

для некоторого $\alpha>1$. Тогда

$$
f(z)=\sum_{0<k<\alpha-1} a_{k} z^{k}+\sum_{1-\alpha<k<0} b_{k}(\bar{z})^{-k}+O(\ln |z|) \quad \text { при } z \rightarrow \infty,
$$

где $a_{k}, b_{k} \in \mathbb{C}$ и суммы с пустым множеством индексов суммирования считаются равНЫми нулю.

ДокАЗАТЕЛЬСтво. Пусть $B$ - открытый круг в $\mathbb{C}$ с центром в нуле, содержащий множество $\bar{G}$. При $z=\rho e^{i \varphi} \in \mathbb{C} \backslash \bar{B}$ имеем равенство

$$
f(z)=\sum_{n=-\infty}^{\infty} f_{n}(\rho) e^{i n \varphi}
$$

где

$$
f_{n}(\rho)=\frac{1}{2 \pi} \int_{0}^{2 \pi} f\left(\rho e^{i \varphi}\right) e^{-i n \varphi} d \varphi .
$$

Из (30) и гармоничности $f$ следует, что ряд (29) сходится локально равномерно в $\mathbb{C} \backslash \bar{B}$ и при этом функции $f_{n}(\rho) e^{i n \varphi}$ гармонические в $\mathbb{C} \backslash \bar{B}$ (см. (3)-(5)). Используя формулу (6), отсюда получаем

$$
f_{0}(\rho)=a_{0}+b_{0} \ln \rho, \quad f_{n}(\rho)=a_{n} \rho^{n}+b_{n} \rho^{-n} \quad \text { при } n \neq 0,
$$

где $a_{n}, b_{n} \in \mathbb{C}$. Равенства $(30),(31)$ и (1) показывают, что

$$
\left|a_{n} r^{n}+b_{n} r^{-n}\right| \leq \frac{M_{r}(f)}{2 \pi r} \quad \text { при } r>0, n \neq 0 .
$$

Поэтому из (28) следует, что $a_{n}=0$ при $n \geq \alpha-1$ и $b_{n}=0$ при $n<1-\alpha$. Отсюда и из соотношений (31) и (29) получаем требуемое утверждение.

ЗАмечАние 1. Далеко идущие обобщения леммы 6 , связанные с поведением на бесконечности решений уравнений свертки на симметрических пространствах, получены в $[18$, гл. 15$]$ (для случая пространства $\mathbb{R}^{n}$ см. также $[2$, ч. 3 , гл. 3]). 


\section{§ 3. Доказательства основных результатов}

ДОКАЗАТЕЛЬСТВО тЕОРЕМЫ 1 . Пусть функция $f$ удовлетворяет условиям теоремы 1. Рассмотрим функцию $u(z)=f(\psi(z))$, где $\psi-$ функция, определенная в $\S 2$. Из условия теоремы 1 получаем, что $u$ гармонична в области $A$ и непрерывна в $\bar{A}$, при этом

$$
u\left(e^{i \varphi}\right)=c_{1} \quad \text { для любого } \varphi \in[0,2 \pi] .
$$

Кроме того, из условия (3) теоремы 1 , равенства (9) и леммы 6 следует, что

$$
u(z)=O(|z|) \quad \text { при } z \rightarrow \infty .
$$

При любом фиксированном $\rho \geq 1$ функции $u\left(\rho e^{i \varphi}\right)$ соответствует ряд Фурье $(3)$, в котором функции $u_{n}(\rho)$ непрерывны на $[1,+\infty)$ (см. (4)). Из гармоничности $u$ и соотношения (5) получаем, что функции $u_{n}(\rho) e^{i n \varphi}$ гармонические в $A$ при всех $n$. В силу (6) это означает, что

$$
u_{0}(\rho)=a_{0}+b_{0} \ln \rho, \quad u_{n}(\rho)=a_{n} \rho^{n}+b_{n} \rho^{-n} \quad \text { при } n \neq 0,
$$

где $\rho \geq 1$ и $a_{n}, b_{n}$ - комплексные постоянные. Из равенств (32) и (4) следует, что $u_{0}(1)=c_{1}$ и $u_{n}(1)=0$ при $n \neq 0$. Следовательно,

$$
a_{0}=0 \quad \text { и } \quad a_{n}+b_{n}=0 \quad \text { при } n \neq 0 .
$$

Далее, из (4) и (33) получаем

$$
u_{n}(\rho)=O(\rho) \quad \text { при } \rho \rightarrow+\infty .
$$

Последнее равенство означает, что $a_{n}=0$ и $b_{-n}=0$ при $n \geq 2$. Сопоставляя это с (34), приходим к выводу, что при $|z| \geq 1$

$$
u(z)=b_{0} \ln |z|+a_{1}\left(z-\frac{1}{\bar{z}}\right)+a_{-1}\left(\frac{1}{z}-\bar{z}\right),
$$

где черта означает знак комплексного сопряжения. Из этого равенства находим

$$
\lim _{\varepsilon \rightarrow+0} \frac{u\left((1+\varepsilon) e^{i \varphi}\right)-u\left(e^{i \varphi}\right)}{\varepsilon}=b_{0}+2 a_{1} e^{i \varphi}-2 a_{-1} e^{-i \varphi}
$$

для любого $\varphi \in[0,2 \pi]$. Отсюда, используя лемму 5 и условие $(2)$ теоремы 1 , заключаем, что

$$
b_{0}+2 a_{1} e^{-i \varphi}-2 a_{-1} e^{i \varphi}=c_{2}\left|h\left(e^{i \varphi}\right)\right|
$$

для почти всех $\varphi \in[0,2 \pi]$. Если $c_{2}=0$, то из последнего равенства следует, что $b_{0}=a_{1}=a_{-1}=0$. В силу (35), это означает, что $f=c_{1}$.

Предположим, что $c_{2} \neq 0$. Тогда из (36) имеем

$$
c_{2}^{-1}\left(b_{0}+2 a_{1} e^{-i \varphi}-2 a_{-1} e^{i \varphi}\right) \geq 0 .
$$

По теореме Фейера - Рисса (см. [25, приложение 5]) неотрицательный тригонометрический полином в левой части равенства (37) можно представить в виде

$$
c_{2}^{-1}\left(b_{0}+2 a_{1} e^{-i \varphi}-2 a_{-1} e^{i \varphi}\right)=\left|\alpha+\beta e^{i \varphi}\right|^{2}, \quad \varphi \in[0,2 \pi],
$$

где комплексные постоянные $\alpha, \beta$ таковы, что

$$
\alpha+\beta z \neq 0 \quad \text { при } z \in \mathbb{D} .
$$


Докажем, что $\beta=0$. Предположим противное, тогда из (39) имеем $|\alpha| \geq|\beta|>0$. Применяя лемму 1 и следствие 3, отсюда заключаем, что функции

$$
h_{1}(z)=\frac{h(z)}{(\alpha+\beta z)^{2}}, \quad h_{2}(z)=\frac{1}{h_{1}(z)}
$$

принадлежат классу $H_{p}(\mathbb{D})$ при $p \in(0,1 / 4)$. Кроме того, из $(36)$ и $(39)$ следует, что

$$
\left|h_{1}\left(e^{i \varphi}\right)\right|=\left|h_{2}\left(e^{i \varphi}\right)\right|=1
$$

для почти всех $\varphi \in(0,2 \pi)$. В силу теоремы В. И. Смирнова (см. [24, гл. 9, $\S 4$, теорема 4]) это означает, что $\left|h_{1}(z)\right|=1$ при всех $z \in \mathbb{D}$. Следовательно, $h(z)=\gamma\left(\alpha^{2}+2 \alpha \beta z+\beta^{2} z^{2}\right)$, где $\gamma \in \mathbb{C},|\gamma|=1$. Учитывая (11), отсюда получаем $\beta=0$, что противоречит нашему предположению. Данное рассуждение и формулы $(36)$ и (38) показывают, что $\beta=a_{1}=a_{-1}=0$ и $\left|h\left(e^{i \varphi}\right)\right|=c_{2}^{-1} b_{0}$ для почти всех $\varphi \in(0,2 \pi)$. Как и выше, отсюда и из теоремы В. И. Смирнова заключаем, что $h$ - тождественная константа. Учитывая (11) и (9), имеем $h(z)=a$ в $\mathbb{D}$ и $\psi(z)=a z+\psi_{0}$ в $A$. Следовательно, область $G$ является кругом с центром $z_{0}=\psi_{0}$ и радиусом $R=a$. В этом случае из равенства (35) получаем соотношение (2), что завершает доказательство теоремы 1.

ДоКАЗАТЕЛЬСТво теОРЕмы 2. Пусть $f$ удовлетворяет условиям теоремы 2. Повторяя рассуждения из доказательства теоремы 1 , получаем, что для функции $u(z)=f(\psi(z))$ выполнены соотношения (32)-(34). Используя лемму 6 и условие $(3)$ теоремы 2 при $\alpha>1$, как и выше, приходим к равенству

$$
u(z)=c_{1}+b_{0} \ln |z|+\sum_{0<k<\alpha-1}\left(a_{k}\left(z^{k}-(\bar{z})^{-k}\right)+a_{-k}\left(z^{-k}-\bar{z}^{k}\right)\right) .
$$

Из этого равенства, леммы 5 и условия (2) теоремы 2 следует, что

$$
b_{0}+2 \sum_{0<k<\alpha-1}\left(a_{k} e^{i k \varphi}-a_{-k} e^{-i k \varphi}\right)=0
$$

для некоторого множества точек $\varphi \in[0,2 \pi]$ положительной лебеговой меры. Это означает, что $b_{0}=0$ и $a_{k}=0$ при $0<|k|<\alpha-1$. Отсюда, из равенства (40) и определения $u$ получаем, что $f=c_{1}$.

ДОКАЗАТЕЛЬСТВо ТЕОРЕмЫ 3. Очевидно, при любом достаточно малом $\varepsilon \in(0,1)$ выполнено неравенство

$$
\frac{2}{3}+\frac{1}{9(1-\varepsilon)^{2}}<(1-\varepsilon)^{2}
$$

Для таких $\varepsilon$ обозначим $A_{\varepsilon}=\{z \in \mathbb{C}:|z|>1-\varepsilon\}$. Рассмотрим функцию

$$
\Phi(z)=z-\frac{2}{3 z}-\frac{1}{27 z^{3}}, \quad z \in A_{\varepsilon} .
$$

Для любых $z_{1}, z_{2} \in A_{\varepsilon}$ имеем оценки

$$
\left|z_{1} z_{2}\right|>(1-\varepsilon)^{2}, \quad\left|z_{1}^{-2}+\left(z_{1} z_{2}\right)^{-1}+z_{2}^{-2}\right|<\frac{3}{(1-\varepsilon)^{2}} .
$$

Кроме того, из (42) находим

$$
\Phi\left(z_{1}\right)-\Phi\left(z_{2}\right)=\left(z_{1}-z_{2}\right)\left(1+\frac{1}{z_{1} z_{2}}\left(\frac{2}{3}+\frac{1}{27}\left(z_{1}^{-2}+\left(z_{2} z_{2}\right)^{-1}+z_{2}^{-2}\right)\right)\right) .
$$


Учитывая неравенства (41) и (43), из последнего соотношения заключаем, что $\Phi\left(z_{1}\right) \neq \Phi\left(z_{2}\right)$ при $z_{1} \neq z_{2}$. Таким образом, $\Phi$ однолистна в области $A_{\varepsilon}$. Положим

$$
\Phi\left(A_{\varepsilon}\right)=\left\{z \in \mathbb{C}: z=\Phi(\zeta), \zeta \in A_{\varepsilon}\right\}
$$

и обозначим через $g$ обратную к $\Phi$ функцию, действующую из $\Phi\left(A_{\varepsilon}\right)$ на $A_{\varepsilon}$. Из формулы (42) следует, что при любом $z \in A_{\varepsilon}$ выполнены неравенства

$$
|z|-\frac{2}{3(1-\varepsilon)}-\frac{1}{27(1-\varepsilon)^{3}}<|\Phi(z)|<|z|+\frac{2}{3(1-\varepsilon)}+\frac{1}{27(1-\varepsilon)^{3}} .
$$

Отсюда и из определения $g$ получаем, что

$$
|z|-\frac{2}{3(1-\varepsilon)}-\frac{1}{27(1-\varepsilon)^{3}}<|g(z)|<|z|+\frac{2}{3(1-\varepsilon)}+\frac{1}{27(1-\varepsilon)^{3}}
$$

при всех $z \in \Phi\left(A_{\varepsilon}\right)$.

Положим $G=\mathbb{C} \backslash \overline{\Phi(A)}$, где $\Phi(A)=\{z \in \mathbb{C}: z=\Phi(\zeta), \zeta \in A\}$. В силу нечетности и однолистности $\Phi$ множество $G$ является центрально-симметричной ограниченной областью с гладкой жордановой границей $\Gamma=\{z \in \mathbb{C}: z=$ $\Phi(\zeta), \zeta \in \mathbb{T}\}$. Кроме того, поскольку

$$
\Phi(1)=-\Phi(-1)=\frac{8}{27} \quad \text { и } \quad \Phi(i)=-\Phi(-i)=\frac{44}{27} i,
$$

область $G$ не является кругом.

Рассмотрим функции

$$
f_{1}=\ln |g|, \quad f_{2}=\frac{10}{9} \ln |g|-\frac{1}{3} \operatorname{Re}\left(\frac{1}{g^{2}}\right), \quad f_{3}=\frac{10}{9} \ln |g|+\frac{1}{6} \operatorname{Re}\left(g^{2}-\frac{1}{g^{2}}\right) .
$$

Из определения $g$ следует, что $g$ голоморфна в $\Phi\left(A_{\varepsilon}\right)$ и не обращается в нуль. Отсюда получаем, что функции $f_{1}, f_{2}, f_{3}$ гармонические в $\mathbb{C} \backslash \bar{G}$ и непрерывны в $\mathbb{C} \backslash G$. Используя также $(44)$, приходим к выводу, что функции $f_{1}, f_{2}, f_{3}$ удовлетворяют всем требованиям теоремы 3.

\section{ЛИТЕРАТУРА}

1. Владимиров В. С. Уравнения математической физики. М.: Наука, 1981.

2. Volchkov V. V. Integral geometry and convolution equations. Dordrecht: Kluwer, 2003.

3. Netuka I., Vesely J. Mean value property and harmonic functions // Classical and modern potential theory and applications. Dordrecht: Kluwer, 1994. P. 359-398.

4. Волчков В. В. Новые теоремы о двух радиусах в теории гармонических функций // Изв. РАН. Сер. мат. 1994. Т. 58, № 1. С. 182-194.

5. Kuznetsov N. Mean value properties of harmonic functions and related topics (a survey) // arXiv:1904.08312v2[math.AP]22.05.2019. 21 p.

6. Hansen W. A Liouville property for spherical averages in the plane // Math. Ann. 2001. V. 319. P. 539-551.

7. Caruzzo Dolcetta I., Cutrì A. On the Liouville property for sublaplacians // Ann. Scuola Norm. Sup. Pisa Cl. Sci. 1997. V. 25, N 4. P. 239-256.

8. Cutrì A., Leoni F. On the Liouville property for fully nonlinear equations // Ann. Inst. Henri Poincaré, Anal. Non Linéaire. 2000. V. 17, N 2. P. 219-245.

9. Chu C. H., Lau A. T. M. Harmonic functions on topological groups and symmetric spaces // Math. Z. 2011. Bd 268. Heft 3-4. S. 649-673.

10. Degtyarev S. P. Liouville Property for solutions of the linearized degenerate thin film equation of fourth order in a halfspace // Result. Math. 2016. V. 70. P. 137-161.

11. Heyer $H$. The Liouville property for harmonic functions on groups and hypergroups // Methods Funct. Anal. Topol. 2017. V. 23, N 1. P. 3-25. 
12. Йон $\Phi$. Плоские волны и сферические средние в применении к дифференциальным уравнениям с частными производными. М.: Изд-во иностр. лит., 1958.

13. Smith J. D. Harmonic analysis of scalar and vector fields in $\mathbb{R}^{n} / /$ Proc. Cambridge Philos. Soc. 1972. V. 72. P. 403-416.

14. Волчков В. В. Проблемы типа Помпейю на многообразиях // Докл. АН Украины. 1993. № 11. C. 9-13.

15. Thangavelu S. Spherical means and CR functions on the Heisenberg group // J. Anal. Math. 1994. V. 63. P. 255-286.

16. Rawat R., Sitaram A. The injectivity of the Pompeiu transform and $L^{p}$-analogues of the Wiener Tauberian theorem // Israel J. Math. 1995. V. 91. P. 307-316.

17. Волчков В. В. Решение проблемы носителя для некоторых классов функций // Мат. сб. 1997. T. 188, № 9. C. 13-30.

18. Volchkov V. V., Volchkov Vit. V. Harmonic analysis of mean periodic functions on symmetric spaces and the Heisenberg group. London: Springer-Verl., 2009.

19. Volchkov V. V., Volchkov Vit. V. Offbeat integral geometry on symmetric spaces. Basel: Birkhäuser, 2013.

20. Еграфов М. А. Асимптотические оценки и целые функции. М.: Физматгиз, 1962.

21. Еграфов М. А. Аналитические функции. М.: Наука, 1965.

22. Shahshahani M., Sitaram A. The Pompeiu problem in exterior domains in symmetric spaces // Contemp. Math. 1987. V. 63. P. 267-277.

23. Волчков В. В., Волчков Вит. В. Поведение на бесконечности решений искаженного уравнения свертки // Изв. РАН. Сер. мат. 2012. Т. 76, № 1. С. 85-100.

24. Голузин Г. М. Геометрическая теория функций комплексного переменного. М.: Наука, 1966.

25. Левин Б. Я. Распределение корней целых функций. М.: Гостехиздат, 1956.

Поступила в редакиию 2 декабря 2019 г.

После доработки 2 декабря 2019 г.

Принята к публикации 19 февраля 2020 г.

Волчков Валерий Владимирович, Волчков Виталий Владимирович

Донецкий национальный университет,

ул. Университетская, 24, Донецк 83001, Украина

valeriyvolchkov@gmail.com, volna936@gmail.com 